\title{
Inducing coherence in networks of bistable maps by varying the interaction range
}

\author{
Pedro G. Lind, ${ }^{1,2,3, *}$ João Corte-Real,,$^{2,3, \dagger}$ and Jason A. C. Gallas ${ }^{1,2,3,4, \sharp}$ \\ ${ }^{1}$ Instituto de Física, Universidade Federal do Rio Grande do Sul, 91501-970 Porto Alegre, Brazil \\ ${ }^{2}$ Unidade de Meteorologia e Climatologia, Instituto de Ciência Aplicada e Tecnologia, Faculdade de Ciências, \\ Universidade de Lisboa, 1749-016 Lisboa, Portugal \\ ${ }^{3}$ Centro de Geofísica, Universidade de Évora, 7000 Évora, Portugal \\ ${ }^{4}$ Institut für Computer Anwendungen, Universität Stuttgart, Pfaffenwaldring 27, D-70569 Stuttgart, Germany
}

(Received 25 August 2003; published 27 February 2004)

\begin{abstract}
Ordinarily, two different topologies have been used to model spatiotemporal chaos and to study complexity in networks of maps: one where sites interact only with nearest neighbors (e.g., the standard diffusive coupling) and one where sites interact with all sites in the network (global coupling). Here we investigate intermediate regimes considering the interaction range as a free tunable parameter. The synchronization behavior normally seen in globally coupled maps is found to set in for interaction ranges considerably smaller than the system size. In addition, we analytically derive stability conditions for the onset of coherent states (full synchronization) from which the minimum interaction range needed to induce coherence in homogeneously coupled maps can be determined. Such conditions are also obtained for inhomogeneous situations when the coupling strength decreases linearly with the distance. The characteristic range for the onset of coherence is studied in detail as a function of model parameters.
\end{abstract}

DOI: 10.1103/PhysRevE.69.026209

PACS number(s): 05.45.Xt, 05.45.Ra, 47.54.+r

\section{INTRODUCTION}

Networks of coupled maps are popular nowadays to model collective behavior of physical systems such as lasers [1], Josephson junction arrays [2], electric circuits [3], connected oscillators [4], and many others [5-8]. Until recently, it was common to use either regular or random networks even though, as is well known, these choices are not adequate to describe all natural phenomena. An interesting way to mitigate this shortcoming is to consider the dynamics of small-world networks [9], namely regular networks with increasing amounts of disorder. These systems can be highly clustered, like regular lattices, yet have small characteristic path lengths, like random graphs [9]. This type of network is a hot topic of research today because it is useful to describe natural phenomena ranging from semiconductor laser arrays [10], patterns in electronic circuits [11], damage spreading in Ising models [12], disease propagation and various epidemiological effects [13-16], and even in sociological phenomena such as rumor spreading and the interactions between networks of acquaintances [17-19].

For regular networks, where space plays a very decisive role, the standard way of investigating complexity is by considering sets of oscillators described locally either with differential equations or with discrete mappings [20]. A very popular model of regular network is the coupled map lattice which, ordinarily, has been used with two different topologies so far. One topology originates from discretizations of differential operators and involves sites which interact only with nearest neighbors, either under the standard diffusive coupling [8] or its diffusive-advective extension [21]. The

\footnotetext{
*URL: http://www.icat.fc.ul.pt/umc/plind

${ }^{\dagger}$ Email address: jcr@fc.ul.pt

¥URL: http://www.if.ufrgs.br/ jgallas
}

second topology involves considering global coupling, when each site receives simultaneous feedback from all other sites in the network through a "mean field."

In the same way as small-world networks explores middle ground between regular and random networks, an interesting open question is to investigate how the interaction range affects collective behavior in dynamical systems and, in particular, how the dynamics evolves as the topology changes from local to global coupling. These questions are important in several applications, in particular in problems involving synchronization in gradient flows and in models of ocean convection [21-23].

The purpose of this paper is to investigate synchronization phenomena in coupled map lattices considering the interaction range as a freely tunable control parameter. In general, two main types of synchronization have been observed [5-7]: full and partial synchronization. Oscillators are said to be fully synchronized when they all have identical amplitudes at each time step, i.e., when they are all in the same state. Fully synchronized states are also called uniform or coherent states. Oscillators are said to be partially synchronized when the system assumes a state composed by several domains, called clusters, each one characterized by a certain number of adjacent oscillators evolving coherently (fully synchronized), but forming altogether a nonuniform state.

Synchronization effects have been reported for global coupling [7,26-30], showing that it is possible to observe transitions from partial to full synchronization and, in addition, coherent states were observed for a wide range of parameter values. For local coupling, additional nontrivial behaviors are observed, e.g., spatiotemporal intermittency [8], pattern selection [8,31], and traveling waves [21,22,31]. Synchronized states may be also found under local coupling $[8,31]$ but only for very specific choices of parameters and/or initial conditions.

The differences observed in the dynamics under both 
types of coupling lead naturally to the question of how coherence arises when tuning between local and global couplings. For instance, is there some threshold of the interaction range beyond which coherence is always observed? How large is this threshold? How does it depend on model parameters? In this paper we answer these questions, showing that it is possible to determine analytically stability conditions for the appearance of coherence states and, from them, to derive interaction range thresholds for coherence. An interesting finding is that transitions from partial to full synchronization occur not only when increasing the coupling strength as reported in the literature, but also when increasing the range of interaction. Furthermore, we find that synchronization behaviors routinely ascribed to maximum interaction range (i.e., to "globally" coupled maps) are in fact observed to set in for significantly shorter interaction ranges, with the system remaining thereafter insensitive to further increase in the number of neighbors.

We start in Sec. II presenting the model used, which allows a separate consideration of both homogeneous and inhomogeneous couplings and for two types of updating of physical importance. In Sec. III we give an illustrative example of a coherent state which appears when the interaction range increases from local coupling. With the help of this example we study numerically the dependence of transitions between nonuniform and coherent states on the local bistability, on the coupling strength and on the lattice size. In Sec. IV we analyze coherent states in detail determining stability conditions as a function of the interaction range, for both types of updating and for homogeneous and inhomogeneous couplings. Our conclusions are presented in Sec. V.

\section{SPATIALLY ISOTROPIC MODEL}

As mentioned above, our goal is to investigate the impact of varying the interaction range in networks of coupled maps. To this end we introduce a convenient control parameter $R$, the "interaction range," specifying the number of neighbors responsible for the feedback in the network. Formally, there are many possible ways to interpolate between local and global coupling. For simplicity we assume neighbors to be symmetrically placed around each site.

As usual, using $\varepsilon$ to represent the coupling strength, 0 $\leqslant \varepsilon \leqslant 1, i=1, \ldots, L$ to represent space ( $L$ is the total number of oscillators) and $t$ to represent a discrete time variable, our spatially isotropic model reads

$$
\begin{aligned}
x_{t+1}(i)= & (1-\varepsilon) f\left(x_{t}(i)\right)+\frac{\varepsilon}{2 R} \sum_{j=1}^{R} W_{R, j}\left[g\left(x_{t}(i+j)\right)\right. \\
& \left.+g\left(x_{t}(i-j)\right)\right],
\end{aligned}
$$

where the interaction range $R$ is an integer number that may vary between 1 and $[(L-1) / 2]$, the integer part of $(L$ $-1) / 2$. In this expression, $f(x)$ rules the local dynamics while $g(x)$ controls interactions among oscillators. These functions contain all nonlinearities of the model and, additionally, $g(x)$ is used to define the type of updating: $g(x)$ $=x$, which we call an updating with linear coupling, and $g(x)=f(x)$, which we call an updating with nonlinear coupling, since $f(x)$ is presumed to be a nonlinear function of $x$. We use periodic boundary conditions.

For $g(x)=x$ the sum in Eq. (1) represents an average over the states of the $R$ nearest neighbors, while for $g(x)=f(x)$ the sum averages their corresponding local oscillations. Therefore, by considering separately both types of updatings, we intend to investigate how these different "mean fields" influence the stability of coherent states. Below we show that although the stability conditions are quite different, the corresponding eigenmodes are qualitatively the same (see Fig. 9 below).

The weights $W_{R, j}$ in Eq. (1) allow one to vary the relative importance of the sites composing the neighborhood and satisfy a normalization which, for later convenience, we write as

$$
\sum_{j=1}^{R} W_{R, j}=R .
$$

The simplest choice $W_{R, j}=1$ for all $j$ provides a homogeneous coupling, by far the most frequently studied situation.

For $R=1$, Eq. (1) reproduces the usual local coupling regime while for $R=[(L-1) / 2]$ it corresponds to the familiar globally coupled maps. The interaction term in Eq. (1) does not contain the amplitudes $x_{t}(i)$ and $x_{t}(L / 2)$ for $L$ even, differently from to the usual model of globally coupled maps [7]. However, numerical results for $R=[L-1] / 2$ are very similar to those observed when using the usual global coupling model [26] and, therefore, $R=[L-1] / 2$ indeed corresponds to this limit. In general, the "interaction term" in Eq. (1), namely the collection of all terms having $\varepsilon$ as coefficient, may be though physically as arising from a discretization of the Laplacian operator averaged over $R$ successive spatial increments.

Variable interaction ranges were considered before. For instance, Chaté and Manneville [32], Zhilin et al. [33], and Carretero-González et al. [34] essentially use equations which may be transformed into Eq. (1) above with $W_{R, j}$ $=1$, while Baptista and Viana [35] use a power-law coupling in the interaction term. These studies considered either nonlinear $[32,35]$ or linear $[33,34]$ coupling updatings. All these works deal with local dynamics ruled by the quadratic map, which is monostable, i.e. allows the presence of just one stable attractor at finite distances.

Motivated by recent applications of cubic maps in the investigation of aspects of ocean convection $[23,36]$, here we also consider cubic local dynamics ruled by the equation

$$
x_{t+1}=f\left(x_{t}\right) \equiv-x_{t}^{3}+a x_{t}+b,
$$

where $a$ and $b$ are real parameters. This map is bistable (has two stable fixed points) and, therefore, allows the investigation of competition between two different phases in the network, as is done in Sec. III. 

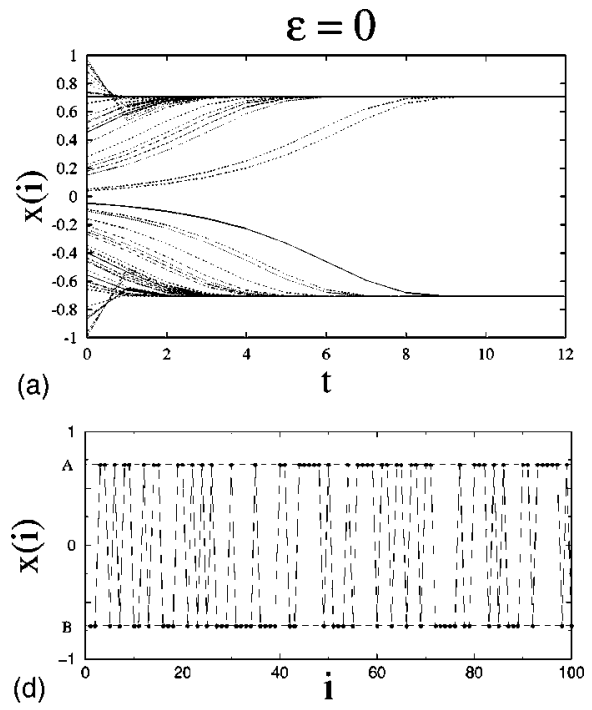

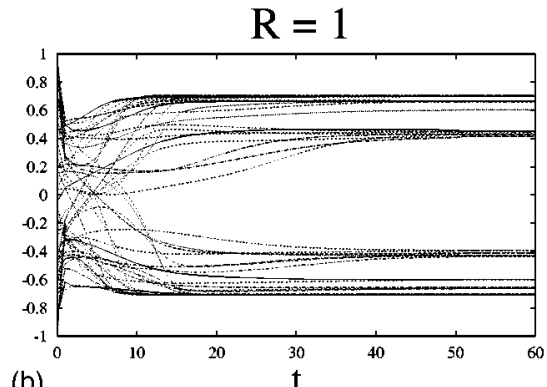

(b)

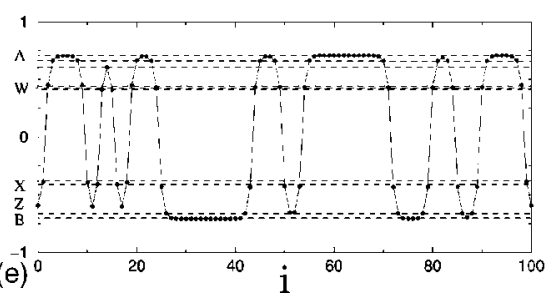

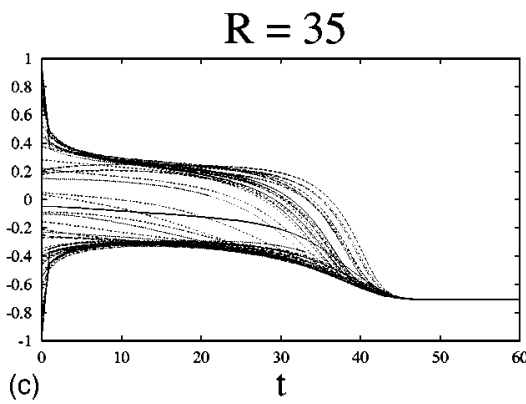

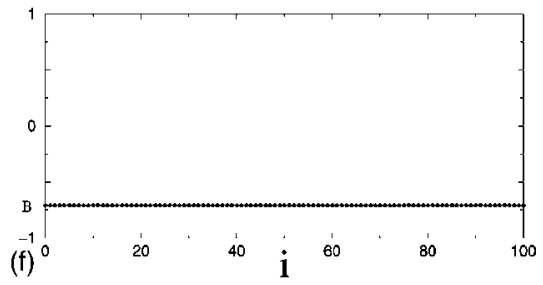

FIG. 1. Upper row: time evolution of the oscillators in a lattice with $L=100$ sites, starting from random initial conditions, homogeneous coupling, linear updating, $a=1.5$ and $b=0$. Three different situations are illustrated: (a) uncoupled regime ( $\varepsilon=0)$, (b) local coupling ( $R$ $=1$ with $\varepsilon=0.3$ ), and (c) coupling beyond the nearest neighbors for $R=35$ also for $\varepsilon=0.3$. Lower row: stationary patterns reached after a transient of 1000 time-steps. Dashed lines indicate admissible local amplitudes, $A, B, \ldots$, for each case.

\section{TWO "ROUTES" TO COHERENCE}

In this section we study the dynamics of the transition from nonuniform to coherent states as a function of the interaction range $R$, under linear updating, $g(x)=x$, and homogeneous coupling, $W_{R, j}=1$. Motivated by the aforementioned application in ocean convection [23], we select the following parameters: $a=1.5$ and $-\beta \leqslant b \leqslant \beta$, where $\beta$ $=\sqrt{6} / 18$, so that the cubic map [Eq. (3)] supports two stable fixed points.

The upper row of Fig. 1 shows the time evolution of 100 individual oscillators while the lower row shows the corresponding spatial configuration after a transient of $10^{4}$ time steps. For clarity, Fig. 1 displays the evolution only 50 of the 100 oscillators. For reference, in the first column one sees the evolution in time and space for the uncoupled case $(\varepsilon$ $=0)$, while the next two columns show the evolution when $\varepsilon=0.3$, for $R=1$ and $R=35$.

For $\varepsilon=0$, when the oscillators are totally uncoupled, their time-evolution must converge towards one of the two possible stable fixed points of Eq. (3). The specific fixed point chosen by each oscillator depends the initial condition. The final spatial configuration of the lattice is composed by a certain number of "clusters" (adjacent oscillators with identical amplitudes [24]), such number being typically of the order of the lattice size. In other words, the uncoupled regime is characterized by a nonuniform (non fully synchronized) state displaying only two possible amplitudes (bistable oscillators).

When the coupling is switched on, neighboring oscillators start to synchronize, decreasing the number of clusters but increasing their sizes. The second column in Fig. 1 illustrates this situation for the same 100 oscillators, this time coupled locally $(R=1)$. From the pattern on the bottom row, one observes that now there are more than just two admissible final amplitudes. In other words, although both stable fixed points $A$ and $B$ remain admissible amplitudes, additional stable amplitudes, say $X, W, Z, \ldots$ appear, as schematically indicated by dashed lines in the figure. Therefore, for short interaction ranges configurations are still nonuniform but display local multistability.

Increasing the number of neighbors $(R>1)$ one observes a gradual decreasing of the number of clusters until the feedback generated by the $2 R$ neighbors is sufficiently strong to induce coherence, i.e., to force all sites towards the same amplitude, as illustrated by the amplitude $B$ in the last column of Fig. 1.

Figure 2, obtained for the same parameters as Fig. 1, displays the fraction $N$ of coherent states as a function of the range, from a sample of 1000 realizations. From this figure two basic facts emerge: (i) the transition from nonuniform to coherent states is rather "smooth": the fraction of initial conditions which converge towards coherence increases essen-

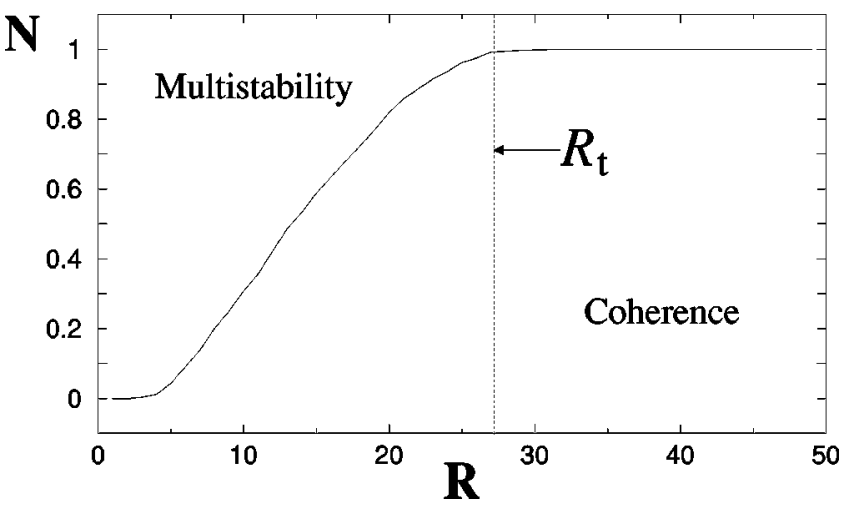

FIG. 2. Fraction of coherent states as a function of the interaction range $R$ over a sample of 1000 sets of random initial conditions, after a transient of $10^{4}$ time-steps. Here $a=1.5, b=0, \varepsilon$ $=0.3$ and $L=100$, as in Fig. 1 . Beyond the threshold $R_{t}$ all oscillators evolve coherently, i.e. $N=1$. 
(a)

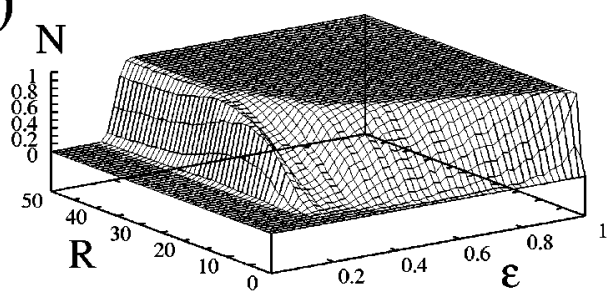

(b)

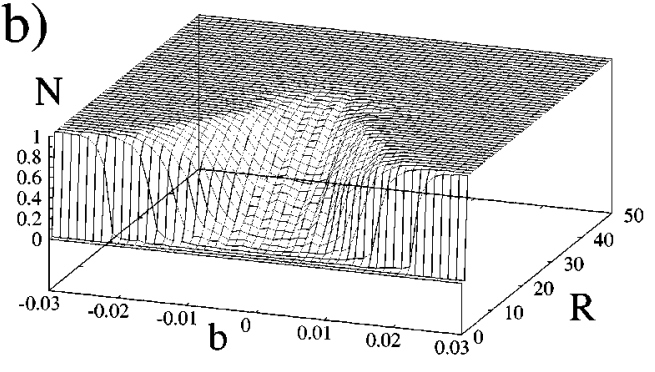

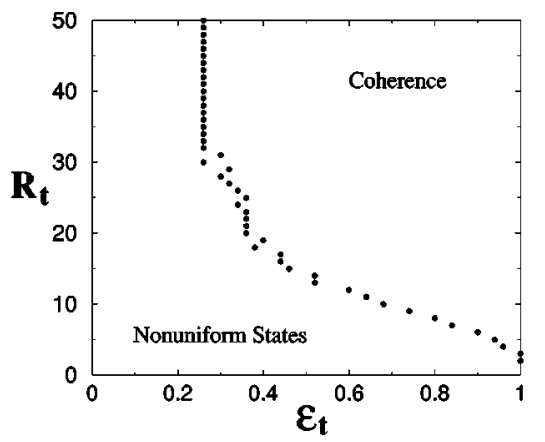

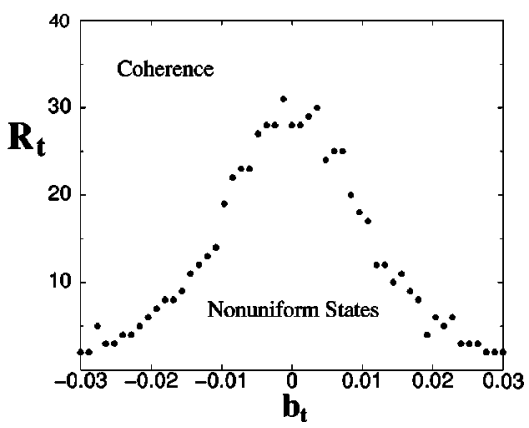

FIG. 3. Fraction $N$ of coherent states as a function of the interaction range $R$ and (a) the coupling strength $\varepsilon$ for $b=0$ and (b) the bistable parameter $b$ for $\varepsilon=0.3$. The values of coherence threshold $R_{t}$ are shown on the right (see the text). The lattice was sampled 500 times and the fraction of coherent states computed after $10^{4}$ time steps. Here $a=1.5$ and $L=100$.

tially linearly with $R(N \propto R)$, and (ii) beyond a characteristic threshold $R_{t}$, all oscillators have the same amplitude and evolve coherently from then on. For the situation depicted in Fig. 2 one has $R_{t}=27$, meaning that the minimum number of neighbors in the interaction term of Eq. (1) to guarantee coherence is $2 R_{t}=54$.

As is clear from Fig. 2, for any range $R \geqslant R_{t}$ all coherence effects usually ascribed to "global" coupling (namely to $R$ $\equiv[(L-1) / 2])$ are already observed. In other words, beyond $R_{t}$ oscillators are totally insensitive to additional feedback from distant neighbors. In particular, $R_{t}$ may be considerably shorter than the lattice size. Notice that it is possible to observe coherent states for $R<R_{t}$. However, the threshold $R_{t}$ is defined here as the range beyond which a coherent state is reached for essentially any choice of initial conditions. In other words, for $R>R_{t}$ the basin of attraction of coherent states essentially fills the entire space of the initial conditions available to the system.

The $R_{t}$ dependence on the coupling strength $\varepsilon$ is shown in the upper row of Fig. 3, where the fraction of coherent states is given on the left as a function of the interaction range and the coupling strength. The corresponding values of $R_{t}$ are shown on the right. As one sees, the threshold $R_{t}$ decreases with the coupling strength and no coherent states are observed, neither for small ranges, $R \leq 5$, nor for small coupling strengths, $\varepsilon \leqq 0.2$.

From Fig. 3 one clearly sees that it is possible to induce coherence by two different ways: by increasing the coupling strength or by increasing the interaction range. The first situation was previously observed in an interesting paper by Zanette [37] while studying a set of coupled differential equations containing a cubic nonlinearity. We now show that this scenario can also be observed in discrete-time models, being one of the two possible scenarios.

Figure 3(b) shows the behavior of $R_{t}$ when the bistability $b$ varies. In the interval $-0.03<b<0.03$, the threshold $R_{t}$ reaches a maximum at $b=0$, decreasing symmetrically with $|b|$. In the rest of the interval $-\beta \leqslant b \leqslant \beta$, not shown in Fig. $3(\mathrm{~b})$, one has $R_{t}=1$ due to the fact that one of the stable fixed points of Eq. (3) has a much larger basin of attraction than the other.

The dependence of $R_{t}$ as a function of both bistability $b$ and diffusion $\varepsilon$ is summarized in Fig. 4, for the same values $a=1.5$ and $L=100$ as before. The symmetry around $b=0$ reflects the symmetry of the basin of the fixed points of the local cubic map (see Fig. 2 of Ref. [23]). For $\varepsilon \leqq 0.2$, one observes that $R_{t}$ is the lattice size $L$ itself $(R \geqslant L / 2)$, while

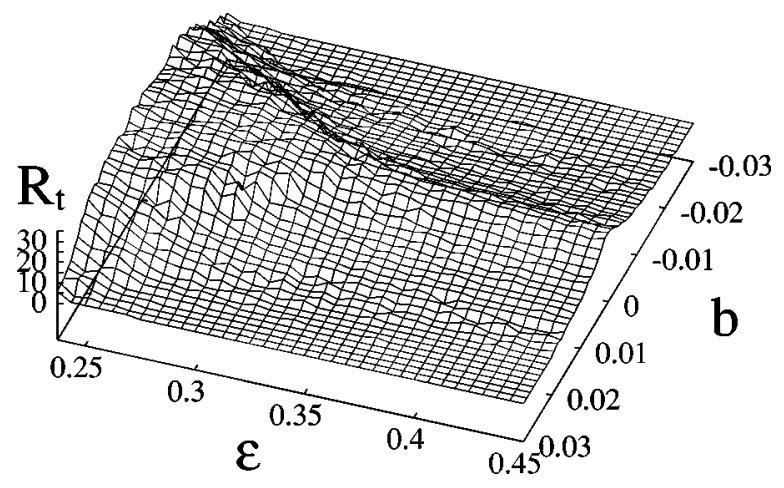

FIG. 4. The threshold $R_{t}$ as a function of $b$ and $\varepsilon$. The cuts at $b=0$ and $\varepsilon=0.3$ are shown in Figs. 3(a) and 3(b), respectively. Here $a=1.5$ and $L=100$ and for each pair $(b, \varepsilon)$ samples of 200 initial conditions were used. 


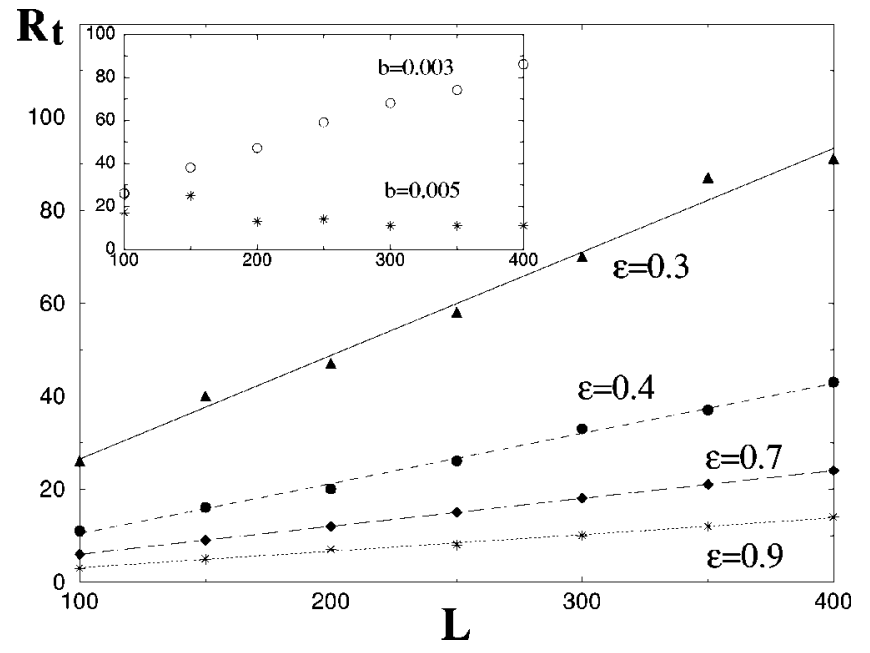

FIG. 5. The threshold $R_{t}$ as a function of the lattice size $L$ for five different coupling strengths, all for $b=0$. The inset shows $R_{t}$ for $b=0.003$ and $b=0.005$, both for $\varepsilon=0.3$. Results from simulations are represented by symbols while the corresponding linear fits are plotted with lines, which have correlation coefficients always greater than 0.993 . Here, $a=1.5$ and a transient of 50000 time steps was used for a sample of 50 sets of initial condition.

for $\varepsilon \gtrsim 0.7$ we find that coherence is already observed for any number of neighbors $\left(R_{t}=1\right)$.

The influence of the lattice size is displayed in Fig. 5, where $R_{t}$ is shown as a function of $L$, for several values of the coupling strength, namely, $\varepsilon=0.3,0.4,0.5,0.7$, and 0.9 , and for $b=0$. From this figure one clearly sees that the threshold $R_{t}$ increases linearly with $L$, while the slope $R_{t} / L$ decreases with $\varepsilon$. Our simulations have shown that the linearity between $R_{t}$ and the lattice size holds only in a neighborhood of $b=0$. An illustration of this fact may be seen in the inset of Fig. 5 where, apart from the different slopes, a linear variation seems to exist for $b=0.003$, but not for $b$ $=0.005$. Once again, the reason for this behavior is that outside the $b=0$ neighborhood, one of the fixed points of the local cubic map has a much larger basin of attraction than the other one and, therefore, almost all sites converge always to the same amplitude even for very short interaction ranges.

So far we have focused in the parameters leading to coherent states without worrying about which precise amplitudes characterize these states. Since multistability is obviously present in the cubic map, being in fact a feature that we want to explore in later applications, in the next section we study specific coherent states supported by the model in Eq. (1) and investigate their stability.

\section{STABILITY OF COHERENT STATES}

In this section we consider homogeneous and inhomogeneous coupling for both types of updatings, $g(x)=x$ and $g(x)=f(x)$, deducing stability conditions for coherent states, reverting the procedure previously used: instead of starting from a random nonuniform state and studying the transition to coherence, we now analytically determine the coherent states, and investigate how they lose stability when varying parameters.
We obtain two different stability conditions, yielding two different scenarios for coherent states to lose their stability: through a period-doubling bifurcation or through a metamorphosis with the stabilization on a nonuniform state. Furthermore, we show that the period-doubling bifurcation is a function of the coupling strength only, while the transition to nonuniform states depends also on the interaction range. The stability conditions obtained are very general, remaining valid for any local dynamics, although they are applied here to the specific cubic map of Eq. (3).

\section{A. Linear and homogeneous coupling}

For homogeneous coupling and linear updating Eq. (1) reduces to

$$
x_{t+1}(i)=(1-\varepsilon) f\left(x_{t}(i)\right)+\frac{\varepsilon}{2 R} \sum_{j=1}^{R}\left[x_{t}(i+j)+x_{t}(i-j)\right] .
$$

Coherent states are obtained from Eq. (4) when substituting $x_{t}(i)$ by a spatially constant amplitude, say $X_{t}$. For coherent states with period 1 , one has only one value $X_{t} \equiv X$ for all time steps, and the stability conditions are determined from the Jacobian matrix of Eq. (4), namely,

$$
\begin{aligned}
\mathbb{J} & =\frac{\partial\left[x_{t+1}(j)\right]}{\partial\left[x_{t}(i)\right]} \\
& =\operatorname{circ}\left((1-\varepsilon) f^{\prime}, \frac{\varepsilon}{2 R}, \ldots, \frac{\varepsilon}{2 R}, 0, \ldots, 0, \frac{\varepsilon}{2 R}, \ldots, \frac{\varepsilon}{2 R}\right),
\end{aligned}
$$

where "circ" indicates the circulant form [38] of the matrix $J$, and $i, j=1, \ldots, L$ and $f^{\prime} \equiv f^{\prime}(X)$ is the first derivative of $f(x)$ computed at the amplitude $X$ of the coherent state. The eigenvalues of $\mathbb{J}$ are [38]

$$
\lambda_{\ell}=(1-\varepsilon) f^{\prime}(X)+\frac{\varepsilon}{2 R} \sum_{j=1}^{R}\left(\omega_{\ell, L}^{j}+\omega_{\ell, L}^{L-j}\right),
$$

where $\ell=1, \ldots, L$, and the coefficients $\omega_{\ell, L}$ represent the $L$ th roots of unity,

$$
\omega_{\ell, L}=\exp \left(i \frac{2 \pi(\ell-1)}{L}\right) .
$$

We now analyze the stability of a period-1 coherent state $X$, evaluating all eigenvalues $\lambda_{\ell}$ in three steps.

First, substituting Eq. (7) into Eq. (6), one obtains a more convenient expression

$$
\lambda_{\ell}=(1-\varepsilon) f^{\prime}(X)+\frac{\varepsilon}{R} \sum_{j=1}^{R} \cos \left(\frac{2 \pi(\ell-1) j}{L}\right) .
$$

For $\ell=1$ the eigenvalue does not depend on $R$ :

$$
\lambda_{1}=(1-\varepsilon) f^{\prime}(X)+\varepsilon
$$


while for all other values of $\ell, 2 \leqslant \ell \leqslant L$, the eigenvalues reduce to

$$
\lambda_{\ell}=(1-\varepsilon) f^{\prime}(X)+\varepsilon \eta(R, L, \ell),
$$

where $\eta(R, L, \ell)$ is given by (see the Appendix)

$$
\eta(R, L, \ell)=\frac{1}{R} \frac{\sin (R \alpha) \cos [(R+1) \alpha]}{\sin \alpha},
$$

where $\alpha=(\ell-1) \pi / L$.

Second, as is well known [25], coherent states always lie on the main diagonal of phase-space being, therefore, proportional to the $L$-dimensional vector $(1, \ldots, 1)$. This vector is the only eigenvector of the Jacobian with eigenvalue $\lambda_{1}$. Thus, assuming that all the other eigenvalues have magnitudes less than 1 , the coherent state with period $\tau=1$ is stable if and only if $\left|\lambda_{1}\right|<1$, yielding

$$
-\frac{1+\varepsilon}{1-\varepsilon}<f^{\prime}(X)<1
$$

The upper limit in Eq. (12) corresponds to the tangent bifurcation of the local map, while the lower limit corresponds to a period-doubling bifurcation, in which a stable coherent state of period $\tau=2$ appears. When $\varepsilon=0$ Eq. (12) reduces to the usual stability condition of the local map, $\left|f^{\prime}(X)\right|<1$. When $\varepsilon \neq 0$, Eq. (12) shows that the size of the interval of stability increases with $\varepsilon$.

Third, the eigenvalues other than $\lambda_{1}$ belong to the manifold transverse to the main diagonal. Thus, the stability condition $\left|\lambda_{\ell}\right| \leqslant 1$ must also hold for all $\ell=2, \ldots, L$, yielding

$$
\xi_{-}(a, b, \varepsilon)<\eta(R, L, \ell)<\xi_{+}(a, b, \varepsilon),
$$

where

$$
\xi_{ \pm}(a, b, \varepsilon)=\left(1-\frac{1}{\varepsilon}\right) f^{\prime}(X) \pm \frac{1}{\varepsilon} .
$$

When $\eta(R, L, \ell)$ crosses one of the two boundaries, $\xi_{+}$or $\xi_{-}$, the coherent states lose stability and a nonuniform state appears.

In short, when Eq. (13) is satisfied, period-1 coherent states are stable in the range defined by Eq. (12) and bifurcate to a period- 2 coherent state when crossing the lower boundary. On the other hand, as long as Eq. (12) holds, the coherent states may only lose stability by "bifurcating" to a nonuniform state when $\eta(R, L, \ell)$ crosses one of the boundaries $\xi_{-}$or $\xi_{+}$in Eq. (13).

Next, we will assume that the condition in Eq. (12) holds, and study the loss of synchronization with the emergence of a nonuniform state, occurring when $\eta(R, L, \ell)=\xi_{+}$or $\eta(R, L, \ell)=\xi_{-}$. It is important to notice that $\eta(R, L, \ell)$ is defined in the interval $[-1,1]$, regardless the lattice size $L$. Therefore, we need to investigate now all possible intervals generated by the conditions $\left|\xi_{ \pm}\right|>1$ and $\left|\xi_{ \pm}\right|<1$. Since $\xi_{-}>-1$ and $\xi_{+}<1$ yields no solutions, altogether there are four cases to be investigated.
(I) When $\xi_{-}>1$ or $\xi_{+}<-1$, coherent states are always unstable, since Eq. (13) is never satisfied. However, this situation never occurs because Eq. (12) must be obeyed.

(II) When $\xi_{-}<-1$ and $\xi_{+}<1$, coherent states are stable as long as $-1<\eta(R, L, \ell)<\xi_{+}(a, b, \varepsilon)$. This situation also never occurs because Eq. (12) must be obeyed.

(III) When $\xi_{-}<-1$ and $\xi_{+}>1$, coherent states are always stable since Eq. (13) is always satisfied. Notice that $\xi_{-}<-1$ and $\xi_{+}>1$ is equivalent to saying that -1 $<f^{\prime}(X)<1$. Thus, in the region where the fixed points of the local dynamics $f(x)$ are stable, coherent states will be stable regardless of the lattice size and interaction range.

(IV) When $\xi_{-}>-1$ and $\xi_{+}>1$, coherent states are stable as long as $\xi_{-}(a, b, \varepsilon)<\eta(R, L, \ell)<1$. This situation occurs for $-(1+\varepsilon) /(1-\varepsilon)<f^{\prime}(X)<-1$. Thus, there is a set of values $(R, L, \ell)$ satisfying

$$
\eta(R, L, \ell)>\left(1-\frac{1}{\varepsilon}\right) f^{\prime}(X)-\frac{1}{\varepsilon},
$$

for which the coherent states are stable, and the threshold $R_{t}$ is the lowest value of $R$ satisfying Eq. (15) for all possible values of $\ell$. Notice that, since $-1 \leqslant \eta \leqslant 1$, it is only necessary to consider $-1 /(1-\varepsilon)<f^{\prime}(X)<-1$, because for the rest of the interval, $-(1+\varepsilon) /(1-\varepsilon)<f^{\prime}(X)<-1 /(1-\varepsilon)$, the condition of Eq. (15) does not hold for any interaction range.

Summarizing, for a given set of parameters $(a, b, \varepsilon, L)$, it is possible to compute analytically the threshold $R_{t}$ where coherence appears. All these considerations are general, independently of the local dynamics $f(x)$. Now we apply the above results to the cubic map, Eq. (3).

For $\varepsilon=1$, a brief analysis shows that coherent states may only have period $\tau=1$ because of the updating. For $\varepsilon \neq 1$, period-1 coherent states are the fixed points of the local cubic map, solutions of $X=f(X)$, namely,

$$
\begin{gathered}
A=\rho+\frac{\theta}{\rho}, \\
B=\omega \rho+\omega^{2} \frac{\theta}{\rho}, \\
C=\omega^{2} \rho+\omega \frac{\theta}{\rho},
\end{gathered}
$$

where $\theta=(a-1) / 3, \rho$ is anyone of the cubic roots of $b / 2$ $+\sqrt{D}$, with $D$ being the discriminant,

$$
D \equiv\left(\frac{b}{2}\right)^{2}+\left(\frac{1-a}{3}\right)^{3},
$$

and $\omega=(-1+i \sqrt{3}) / 2$.

Coherent states must have real values and, therefore, if $D>0$ then only $A$ is real while $B$ and $C$ are complex conjugate, implying existence of only one coherent state. If $D$ $\leqslant 0$, then all three solutions are real. For $D=0$ one finds two different coherent states, $A$ and $B=C$. Figure 6(a) shows these three regions, $D<0, D=0$, and $D>0$, as functions of 

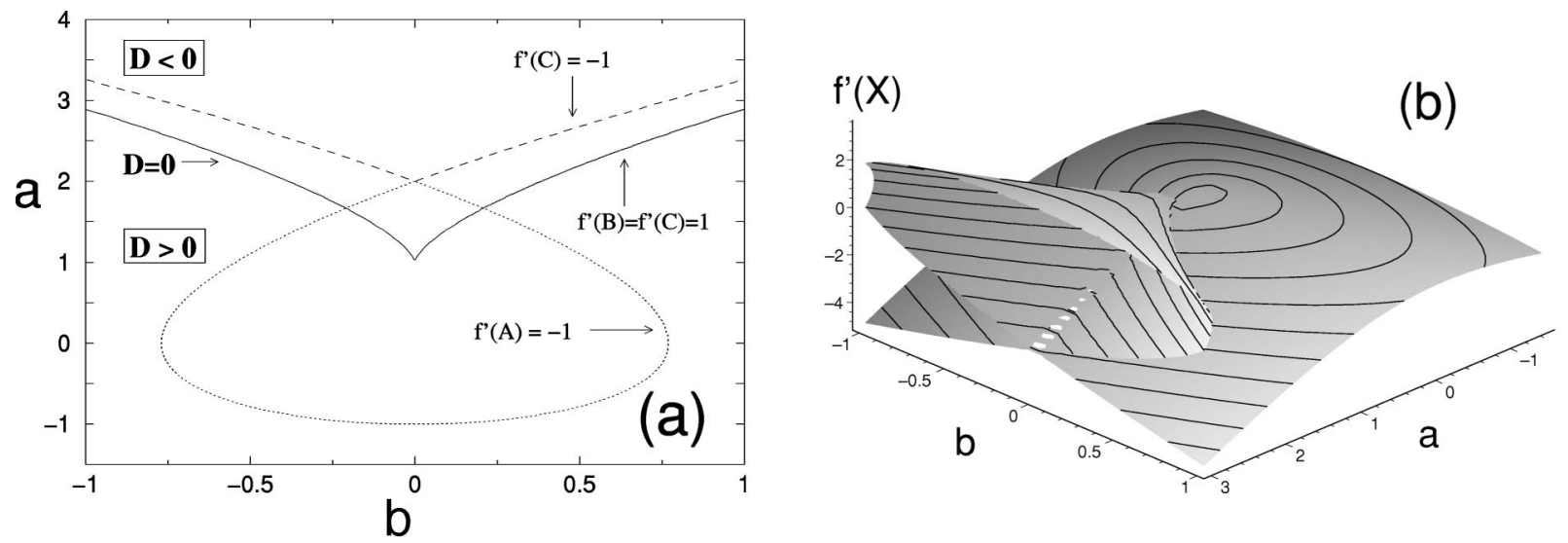

FIG. 6. Stability regions of coherent states in networks of coupled bistable maps. (a) Fixed points of the cubic map, Eq. (3), defining the three possible coherent states, $A, B$ and $C$, given in Eq. (16). Solid line $D=0$ separates two regions: (i) $D>0$ with only one coherent state $A$ and (ii) $D<0$ with three coherent states, $A, B$ and $C$. The solid line also indicates the boundary where tangent bifurcation occurs for $B$ and $C$. For $A$ the tangent bifurcation occurs only at the cusp $(a, b)=(1,0)$. Dotted and dashed lines represent the lines where period-doubling bifurcation occurs for $A$ and $C$, respectively. For the coherent state $B$ no period doubling occurs, i.e., $f^{\prime}(X) \neq-1$ always, as can be seen from (b) where $f^{\prime}(X)$ is plotted for $X=A, B$, and $C$, whenever they are real.

$a$ and $b$. The degenerate case $\rho=0$ (i.e., $D=0$ and $b=0$ ), not included in Eq. (16), yields $A=B=C=0$, corresponding to a cusp at $(a, b)=(1,0)$. The cusp can be easily observed in Fig. 6(b), where the three-dimensional plot shows the value of $f^{\prime}$ of each coherent state, whenever real. Figure 6(a) also shows the bifurcations lines where $\left|f^{\prime}\right|=1$ for each coherent state.

So far, we determined period- 1 coherent states. To conclude this section we now study higher period-doubling assuming, as before, that Eq. (13) holds.

When Eq. (13) holds, coherent states are defined by a period- $\tau$ orbit, $\left\{X_{1}, \ldots, X_{\tau}\right\}$. Substituting these amplitudes into Eq. (4) we obtain a one-dimensional map for coherent states, namely,

$$
X_{t+1}=-(1-\varepsilon) X_{t}^{3}+[\varepsilon(1-a)+a] X_{t}+(1-\varepsilon) b,
$$

with $t=1, \ldots, \tau$. Equation (18) clearly shows that the amplitudes composing periodic orbits of coherent states are always independent of the interaction range.

Figure 7 shows stability domains in parameter space for coherent states observed for three different coupling strengths, $\varepsilon=0,0.1$ and 0.3 . The aforementioned increase of the stability interval as $\varepsilon$ increases, may be explicitly seen in Fig. 7. As already stated, this increase can be explained just by looking at the stability condition in Eq. (12): the period doubling occurs at the lower boundary, which decreases with $\varepsilon$, increasing the range of stability. This increase is seen not only for period-1, has determined analytically above, but also for higher periods.

If one moves to negative values of the coupling strength, the stability regions shrink, until they eventually disappear. Figure 8 shows stability regions for the same periodic orbits as in Fig. 7, but now as a function of $b$ and $\varepsilon$ for $a=1.5$. Clearly, the increasing of $\varepsilon$ to positive values expands the boundaries of the region of bistability. The variation in the full interval $-1 \leqslant \varepsilon \leqslant 1$, incorporates both physical $(0 \leqslant \varepsilon$ $\leqslant 1)$ and non-physical solutions $(-1 \leqslant \varepsilon<0)$. For $\varepsilon=0$, the periodic orbits are stable in a region comprehended between two period-doubling bifurcations, one for positive $b$ and another for the symmetrical negative value (see, e.g., Fig. 2 in Ref. [23]).

One observes a shrinking of the stability regions when $\varepsilon$ decreases towards negative values. In particular, for $\tau=1$
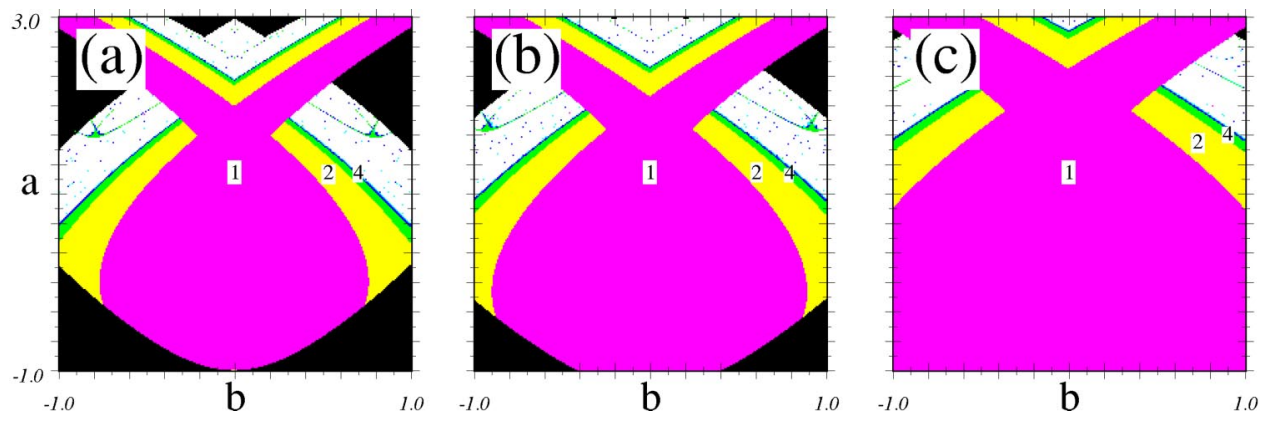

FIG. 7. (Color online) The coupling parameter behaves as an expanding factor of the stability regions of periodic orbits. Illustrative examples for periodic orbits of coherent states with periods $\tau=1,2,4,8$ and 16 for (a) $\varepsilon=0$, (b) $\varepsilon=0.1$, and (c) $\varepsilon=0.3$. Other orbits with higher period $\tau>16$ are indicated in white while the black region represents the attractors $\pm \infty$. Appropriate initial conditions are chosen for each illustration and 1000 transients were used. 


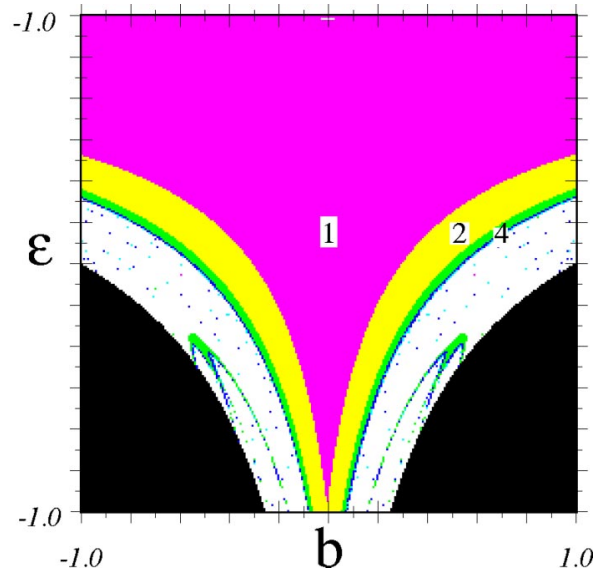

FIG. 8. (Color online) The expansion of the stability regions when the coupling strength increases. Here $a=1.5$. Only the region $0 \leqslant \varepsilon \leqslant 1$ corresponds to "physical" solutions (see the text).

coherent states completely disappear for $\varepsilon \leqslant-1$. Other higher periods disappear also, but at lower coupling strengths. For negative coupling strengths, Fig. 8 shows also that new stability "islands" are found, which do not exist for $\varepsilon \geqslant 0$. In all cases stability regions are symmetric about $b$ $=0$.

This concludes our description of the linear and homogeneous model. We now proceed to consider more realistic situations.

\section{B. Nonlinear and inhomogeneous coupling}

For nonlinear coupling updating, $g(x)=f(x)$, all sites are first updated, $x_{t} \rightarrow f\left(x_{t}\right)$, and then the coupling is considered, namely,

$$
\begin{aligned}
x_{t+1}(i)= & (1-\varepsilon) f\left(x_{t}(i)\right)+\frac{\varepsilon}{2 R} \sum_{j=1}^{R} W_{R, j}\left[f\left(x_{t}(i+j)\right)\right. \\
& \left.+f\left(x_{t}(i-j)\right)\right] .
\end{aligned}
$$

Here, coherent states of any period $\tau$ are always given by the corresponding period- $\tau$ orbits of the uncoupled local map $f(x)$, solutions of $X=f^{(\tau)}(X)$. In particular, for $\tau=1$ we obtain solutions $A, B$, and $C$ as before [see Eqs. (16a)(16c)], but with different stability conditions.

When for all $j$ one takes $W_{R, j}=1$, the eigenvalues of the Jacobian matrix corresponding to Eq. (19) are

$$
\begin{gathered}
\lambda_{1}=f^{\prime}(X), \\
\lambda_{\ell}=f^{\prime}(X)[1-\varepsilon+\varepsilon \eta(R, L, \ell)], \quad \ell=2, \ldots, L .
\end{gathered}
$$

The eigenvalue $\lambda_{1}$ controlling the stability along the main diagonal equals the eigenvalue of the fixed point of the map $f(x)$. The remaining eigenvalues $\lambda_{\ell}$ have eigenvectors in the manifold transverse to the main diagonal, and their dependence on the interaction range $R$ and on the diffusion $\varepsilon$ is illustrated in Fig. 9 (first row).

Thus, on one hand, the coherent state is stable along the main diagonal if and only if the eigenvalue of the local cubic map has absolute value less than unity, with bifurcations occurring when $\left|f^{\prime}(x)\right|=1$. On the other hand, the stability condition in the manifold transverse to the main diagonal is $\left|\lambda_{\ell}\right|<1$ yielding, for all $\ell=2, \ldots, L$,

$$
1-\frac{1}{\varepsilon}-\frac{1}{\varepsilon\left|\lambda_{1}\right|}<\eta(R, L, \ell)<1-\frac{1}{\varepsilon}+\frac{1}{\varepsilon\left|\lambda_{1}\right|},
$$

i.e., $\eta(R, L, \ell)$ should be defined in a range centered at 1 $-1 / \varepsilon$ with amplitude inversely proportional to the eigenvalue $\lambda_{1}$ along the main diagonal. Obviously, here we must have $\varepsilon \neq 0$, otherwise the case is degenerate, with all $L$ eigenvalues being equal to $\lambda_{1}$ [see Eq. (20b)], as expected.

Using a similar analysis as in Sec. IV A one finds that Eq. (21) is satisfied as long as $\left|\lambda_{1}\right|<1$. Thus, for homogeneous coupling with nonlinear updatings, period- $\tau$ coherent states are stable in the same range of parameters as the corresponding period- $\tau$ orbit of the local map $f(x)$. Figure 7(a) illustrates the region of stability for the cubic map in Eq. (3).

Now we proceed to the case of inhomogeneous coupling. This is a more realistic model since space and intersite distance play an important role in the coupling between neighbors. An interesting case, for which we are able to obtain exact analytical results, is that when the coupling strength decreases linearly with the intersite distance, namely,

$$
W_{R, j}=\frac{2(R+1-j)}{R+1} .
$$

In this case period- $\tau$ coherent states are still the $\tau$-periodic orbits of the uncoupled local map but their Jacobian matrix is more complicated. Similarly as in Sec. IV A [see Eq. (8)] we find

$$
\begin{aligned}
\lambda_{\ell}= & f^{\prime}(X)\left[1-\varepsilon+\frac{\varepsilon}{R} \sum_{j=1}^{R} \cos \left(\frac{2 \pi(\ell-1) j}{L}\right)\right. \\
& \left.-\frac{2 \varepsilon}{R(R+1)} \sum_{j=1}^{R} j \cos \left(\frac{2 \pi(\ell-1) j}{L}\right)\right],
\end{aligned}
$$

where $\ell=1, \ldots, L$. For $\ell=1$ one finds $\lambda_{1}=(1-\varepsilon) f^{\prime}(X)$, responsible for the stability along the main diagonal. For $\ell$ $=2, \ldots, L$, the sums of cosines in Eq. (23) may be simplified (see the Appendix) to

$$
\lambda_{\ell}=f^{\prime}(X)[1+\varepsilon \zeta(R, L, \ell)],
$$

where 

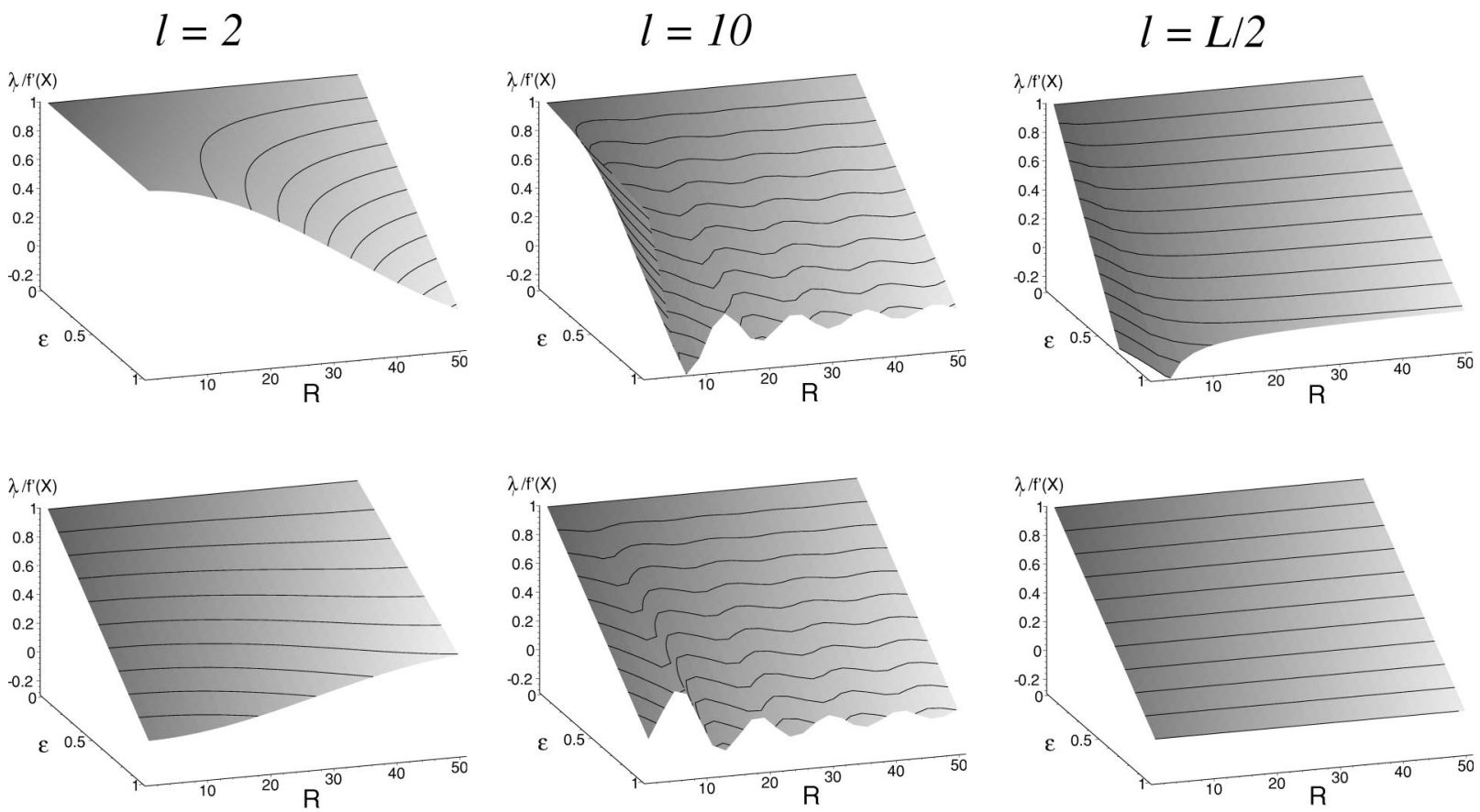

FIG. 9. Representative examples of the eigenvalues $\lambda_{\ell}$ of the manifold transverse to the main diagonal of phase space, as functions of the interaction range $R$ and diffusion $\varepsilon$. First row: homogeneous coupling, Eq. (20b). Second row: inhomogeneous coupling, Eq. (24). Here $L=100$.

$$
\zeta(R, L, \ell)=\frac{1-\cos (2 R \alpha)+2 \sin \alpha[(R+1) \sin (R \alpha) \cos ((R+1) \alpha)-R \sin ((2 R+1) \alpha)-R(R+1) \sin \alpha]}{R(R+1)[1-\cos (2 \alpha)]} .
$$

Similarly to $\eta(R, L, \ell)$, one obtains that $\zeta(R, L, \ell)$ is defined in the interval $-3 / 2<\zeta(R, L, \ell)<4$. Therefore, the corresponding transition thresholds $R_{t}$ may be easily obtained following the same steps above. The second row in Fig. 9 illustrates how the eigenvalues $\lambda_{\ell}$ in Eq. (24) depend on the interaction range $R$ and on the diffusion $\varepsilon$.

For any $W_{R, j}$, nonlinear updatings have always the eigenvalue along the main diagonal of phase space equal to the eigenvalue, $f^{\prime}(X)$, of the uncoupled local cubic map. Furthermore, the eigenvalues of the Jacobian matrix of Eq. (19) can be regarded, in general, as a Fourier sum where the weights $W_{R, j}$ play the role of Fourier coefficients.

\section{CONCLUSIONS}

In this paper we investigated the dynamics of networks of maps with connectivities lying between the usual local coupling, when the neighborhood is the smallest possible, and global coupling, when the neighborhood is maximal.

We find two different ways of inducing coherence: by tuning the coupling strength or by enlarging the interaction range. One interesting finding observed when enlarging the interaction range is that coherent states and coherence phenomena in general, normally observed in networks of globally coupled maps, sets in for connectivities considerably smaller than the network size. In particular, it is possible to define a threshold $R_{t}$ above which full synchronization is always observed, indicating that beyond this threshold each oscillator is not influenced by feedback from additional neighbors.

We also obtained exact stability conditions for the emergence of coherent states in several physical situations of interest, namely, for homogeneous and inhomogeneous coupling under either linear or nonlinear updating. In these situations, coherent states may lose stability through a period-doubling bifurcation which depends only on the coupling strength, or by stabilization on a nonuniform state, depending not only on the coupling strength but also on the interaction range.

The minimal interaction range $R_{t}$ was determined analytically and investigated as a function of nonlinearities and coupling. The stability conditions derived are valid for any local dynamics, either mono or multistable. Of course, for chaotic local dynamics, Jacobians vary in time, and therefore Lyapunov analysis must be used to ascertain the stability of homogeneous states.

This paper considered only networks of identical oscillators. For a number of applications [23], it would be interesting to extend the present study to models containing nonidentical oscillators since local heterogeneity in the network allows modeling additional phenomena such as, e.g., hyster- 
etic behavior. For nonidentical oscillators, it would also be interesting to study the "routes" to coherence reported here for other types of synchronization, such as phase synchronization, and compare them with other studies, e.g., that by Osipov and Kurths [39], where soft and hard transitions to phase synchronization have been found in a lattice of coupled nonidentical circle maps. Since transitions between nonuniform and coherent states may be considered as particular cases of transitions between two different cluster solutions, it would also be of great interest to investigate the generic stability of nonuniform states. We intend to investigate such questions in a subsequent publication.

\section{ACKNOWLEDGMENTS}

P.G.L. thanks Fundação para a Ciência e a Tecnologia, Portugal, for supporting several stays in Brazil during the last few years. This work was part of a Brazilian-Portuguese (CAPES-GRICES) bilateral cooperation.

\section{APPENDIX}

In this appendix we simplify the sum of cosines used in Eqs. (8) and (23). Calling $\alpha=\pi(\ell-1) / L$ and representing cosines by exponentials one finds

$$
\begin{aligned}
\sum_{j=1}^{R} \cos (2 \alpha j) & =\operatorname{Re}\left[\sum_{j=1}^{R} \mathrm{e}^{2 \alpha i j}\right] \\
& =\operatorname{Re}\left[\mathrm{e}^{2 \alpha i} \frac{1-\mathrm{e}^{2 \alpha i R}}{1-\mathrm{e}^{2 \alpha i}}\right] \\
& =\operatorname{Re}\left[\mathrm{e}^{i(R+1) \alpha} \frac{\sin (R \alpha)}{\sin \alpha}\right] \\
& =\frac{\sin (R \alpha) \cos [(R+1) \alpha]}{\sin \alpha} .
\end{aligned}
$$

Dividing by $R$ one obtains Eq. (11). Similarly,

$$
\sum_{j=1}^{R} \sin (2 \alpha j)=\operatorname{Im}\left[\sum_{j=1}^{R} \mathrm{e}^{2 \alpha i j}\right]=\frac{\sin (R \alpha) \sin [(R+1) \alpha]}{\sin \alpha} .
$$

Since

$$
\begin{aligned}
\sum_{j=1}^{R} j \cos (2 \alpha j) & \\
= & \cos (2 \alpha)+2 \cos (2 \times 2 \alpha)+\cdots+R \cos (R 2 \alpha) \\
= & \sum_{j=1}^{R} \cos (2 \alpha j)+\sum_{j=1}^{R-1} \cos [2 \alpha(j+1)] \\
& +\cdots+\sum_{j=1}^{1} \cos [2 \alpha(j+R-1)] \\
& \quad \sum_{k=0}^{R-1} \sum_{j=1}^{R-k} \cos [2 \alpha(j+k)]
\end{aligned}
$$

repeating once again the simplifications done above we find

$$
\begin{aligned}
\sum_{k=0}^{R-1} \sum_{j=1}^{R-k} \cos [2 \alpha(j+k)] \\
=\operatorname{Re}\left[\sum_{k=0}^{R-1} \sum_{j=1}^{R-k} \mathrm{e}^{2 \alpha(j+k) i}\right] \\
=\frac{\sin (R \alpha)}{\sin \alpha} \operatorname{Re}\left[\mathrm { e } ^ { i \alpha ( R + 1 ) } \left(\frac{R}{2}+\frac{1}{2} \sum_{k=0}^{R-1} \cos (2 \alpha k)\right.\right. \\
\left.\left.\quad+i \frac{1}{2} \sum_{k=0}^{R-1} \sin (2 \alpha k)\right)\right] \\
\quad-\frac{\cos (R \alpha)}{\sin \alpha} \operatorname{Re}\left[\mathrm { e } ^ { i \alpha ( R + 1 ) } \left(i \frac{R}{2}+\frac{1}{2} \sum_{k=0}^{R-1} \sin (2 \alpha k)\right.\right. \\
\left.\left.\quad-i \frac{1}{2} \sum_{k=0}^{R-1} \cos (2 \alpha k)\right)\right]
\end{aligned}
$$

Finally, substituting Eqs. (A1) and (A2) into Eq. (A4) above, one obtains

$$
\sum_{j=1}^{R} j \cos (2 \alpha j)=\frac{2 R \sin [(2 R+1) \alpha] \sin \alpha-1+\cos (2 R \alpha)}{2[1-\cos (2 \alpha)]} .
$$

This equation may also be obtained from the derivative of Eq. (A2) with respect to $\alpha$.
[1] A. Uchida, T. Matsuura, S. Kinugawa, and S. Yoshimori, Phys. Rev. E 65, 066212 (2002); J.R. Terry, K.S. Thornburg, Jr., D.J. DeShazer, G.D. VanWiggeren, S. Zhu, P. Ashwin, and R. Roy, ibid. 59, 4036 (1999).

[2] L. Faoro, J. Siewert, and R. Fazio, Phys. Rev. Lett. 90, 028301 (2003); J.A. Acebrón, A. Perales, and R. Spigler, Phys. Rev. E 64, 016218 (2001).

[3] D.A. Smirnov, B.P. Bezruchko, and Y.P. Seleznev, Phys. Rev. E 65, 026205 (2002); G. de Vries, ibid. 64, 051914 (2001).

[4] M. Barahona and L. Pecora, Phys. Rev. Lett. 89, 054101
(2002).

[5] S. Strogatz, Sync: The Emerging Science of Spontaneous Order (Hyperion, New York, 2003).

[6] A. Pikovsky, M. Rosenblum, and J. Kurths, Synchronization-A Universal Concept in Nonlinear Sciences (Cambridge University Press, Cambridge, 2001).

[7] S. Boccaletti, J. Kurths, G. Osipov, D.L. Valladares, and C.S. Zhou, Phys. Rep. 366, 1 (2002).

[8] K. Kaneko and I. Tsuda, Complex Systems: Chaos and Beyond (Springer-Verlag, New York, 2000); Theory and Applications 
of Coupled Map Lattices, edited by K. Kaneko (Wiley, Chichester, 1993).

[9] D.J. Watts and S.H. Strogatz, Nature (London) 393, 440 (1998); S.H. Strogatz, ibid. 410, 268 (2001).

[10] A.G. Vladimirov, G. Kozyreff, and P. Mandel, Europhys. Lett. 61, 613 (2003).

[11] R.F. Cancho, C. Janssen, and R.V. Solé, Phys. Rev. E 64, 046119 (2001).

[12] P. Svenson and D.A. Johnston, Phys. Rev. E 65, 036105 (2002)

[13] M.E.J. Newman, I. Jensen, and R.M. Ziff, Phys. Rev. E 65, 021904 (2002).

[14] M. Kuperman and G. Abramson, Phys. Rev. Lett. 86, 2909 (2001).

[15] N. Zekri and J.P. Clerc, Phys. Rev. E 64, 056115 (2001).

[16] F. Bagnoli and M. Bezzi, Phys. Rev. E 64, 021914 (2001).

[17] J. Davidsen, H. Ebel, and S. Bornholdt, Phys. Rev. Lett. 88, 128701 (2002).

[18] D.H. Zanette, Phys. Rev. E 65, 041908 (2002).

[19] V. Latora and M. Marchiori, Phys. Rev. Lett. 87, 198701 (2001).

[20] M.C. Cross and P.C. Hohenberg, Rev. Mod. Phys. 65, 851 (1993).

[21] P.G. Lind, J. Corte-Real, and J.A.C. Gallas, Phys. Rev. E 66, 016219 (2002).

[22] P.G. Lind, J. Corte-Real, and J.A.C. Gallas, Physica D 168169, 93 (2002).

[23] P.G. Lind, S. Titz, T. Kuhlbrodt, J. Corte-Real, J. Kurths, J.A.C. Gallas, and U. Feudel, Int. J. Bifurcation Chaos, Appl. Sci. Eng. (to be published).
[24] Our definition of a "cluster" is that of Kaneko [8]: a cluster is a set of adjacent oscillators $i_{m} \leqslant i<i_{M}$ such that $\mid x_{t}(i)-x_{t}(i$ $+1) \mid<\delta$ for small $\delta$. Numerically, we used $\delta=0.001$. Although in some situations the above definition was found to lead to spurious results [25], we checked that it is adequate in our case.

[25] A. Pikovsky, O. Popovych, and Y. Maistrenko, Phys. Rev. Lett. 87, 044102 (2001).

[26] K. Kaneko, Phys. Rev. Lett. 63, 219 (1989); 63, 1029 (1989).

[27] S.C. Manrubia and A.S. Mikhailov, Phys. Rev. E 60, 1579 (1999).

[28] T. Shinbrot, Phys. Rev. E 50, 3230 (1994).

[29] M.S. Vieira and A.J. Lichtenberg, Phys. Rev. E 56, R3741 (1997).

[30] F.H. Willeboordse, Phys. Rev. E 65, 026202 (2002).

[31] P.G. Lind, J.A. Corte-Real, and J.A.C. Gallas, Int. J. Bifurcation Chaos Appl. Sci. Eng. 11, 2647 (2001).

[32] H. Chaté and P. Manneville, Chaos 2, 307 (1992).

[33] Q. Zhilin, H. Gang, M. Benkun, and T. Gang, Phys. Rev. E 50, 163 (1994).

[34] R. Carretero-González, D.K. Arrowsmith, and F. Vivaldi, Phys. Rev. E 61, 1329 (2000).

[35] A.M. Baptista and R.L. Viana, Phys. Lett. A 286, 134 (2001).

[36] P.G. Lind, J. Corte-Real, and J.A.C. Gallas, Physica A 327, 65 (2003).

[37] D.H. Zanette, Phys. Rev. E 55, 5315 (1997).

[38] See, e.g., P.J. Davis, Circulant Matrices (Wiley, New York, 1979).

[39] G.V. Osipov and J. Kurths, Phys. Rev. E 65, 016216 (2002). 